\title{
Enfermedad reumática y tratamiento abreviado con corticoides
}

Dres. I. Lema $C^{*}$, L. Sadavedra $M^{*}$., G. Martínez del $\mathrm{R}^{* *}$.

La Enfermedad Reumática sigue siendo una entidad clínica de gran magnitud y una de las principales causas de hospitalizaciones prolongadas en los Servicios de Segunda Infancia en nuestro país.

Como causa de muerte en Chile es responsable de 6 muertes por cada 100.000 habitantes, para el período 1970-1974. De los egresos anuales de las Unidades de Segunda Infancia de los Hospitales Pediátricos de Santiago un 6 a 8\% correspondieron a Enfermedad Reumática para el período 1974-1976. $(1,2)$

De ahí la importancia del uso del esquema abreviado con corticoides en su tratamiento, que reduce en forma significativa el promedio de días de hospitalizacion, sin que esto signifique un riesgo para el niño. ${ }^{(3,4,5,6,7)}$

Hemos revisado todo el material de Enfermedad Reumática desde la creación de este Hospital, a fin de establecer diferencias entre los pacientes hospitalizados antes de junio de 1973 y después de esta fecha, época en que se inició el uso del esquema de tratamiento abreviado en nuestro Servicio.

\section{MATERIAL Y METODO}

Se revisan 150 fichas clínicas clasificadas como Enfermedad Reumática entre noviembre de 1969 y junio de 1977, de ellas 72 casos correspondieron a Enfermedad Reumática Brote Inicial según el criterio de Jones modificado.

Se analiza la distribución de casos por: años, sexo, edad del primer brote, diagnóstico de ingreso, cuadro clínico, exámenes de laboratorio, \footnotetext{
huano.

* Médico Jefe Unidad Segunda Infancia Pediatria Hospital Higueras.
}

* Médico Becario Pediatrín Hospital Higueras, Talca- complicaciones, secuelas y recaídas en relación con el tratamiento efectuado. Además se analizó el promedio de días de hospitalización y su relación con el tratamiento esteroidal, el control posterior al alta y el tratamiento profiláctico.

Como esquema de tratamiento esteroidal abreviado se catalogaron todos aquellos casos en que se usó Prednisona $2 \mathrm{mg}$ por kg. en una dosis diaria administrada a las $08 \mathrm{hr}$. durante 10 días con interrupción brusca. ${ }^{(3,7)}$

\section{RESULTADOS}

Analizando la distribución de casos por años se observó una reducción importante del número de casos a partir del año 1974. Se presentaron 39 casos antes de junio de 1973 y 33 casos posteriores. Debemos hacer notar que el año 1969 se analizó a partir del mes de noviembre. (Gráfico 1.)

\section{Gráfico № 1 \\ ENFERMEDAD REUMATICA DISTRIBUCION POR AÑOS}

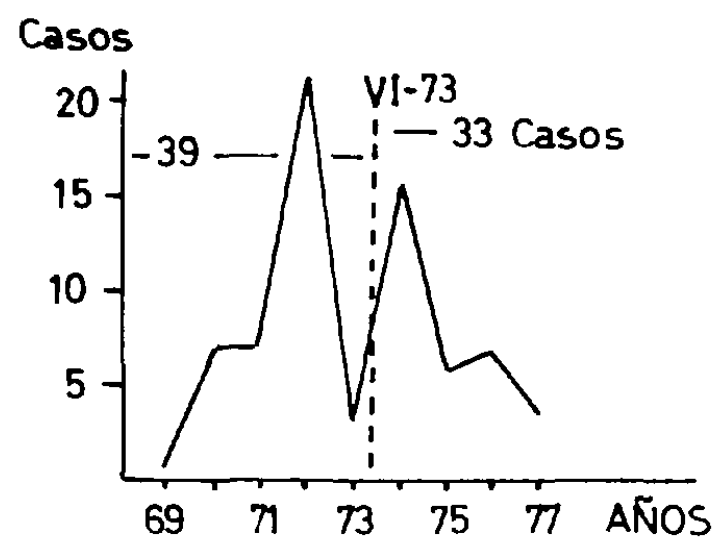


No hubo diferencia significativa en la distribución por sexo con un $48.7 \%$ del sexo femenino y $51.3 \%$ del sexo masculino. El $91.6 \%$ de nuestros pacientes presentaron el primer brote a los 5 años o más. (Gráfico 2.)

\section{Gräfico $\mathrm{N}^{\circ} 2$}

\section{DISTRIBUCION POR SEXO Y EDAD $1{ }^{\circ}$ BROTE}

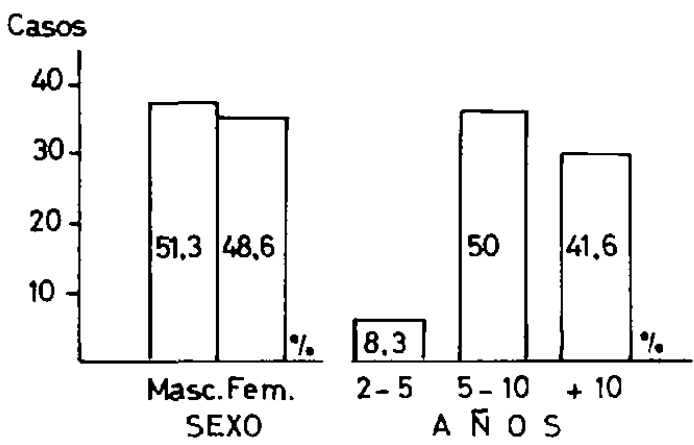

En 63 casos el diagnóstico de ingreso fue Enfermedad Reumática u Obs. Enfermedad Reumática con un $87.4 \%$. (Gráfico 3.)

\section{Gráfico № 3}

\section{DIAGNOSTICO DE INGRESO}

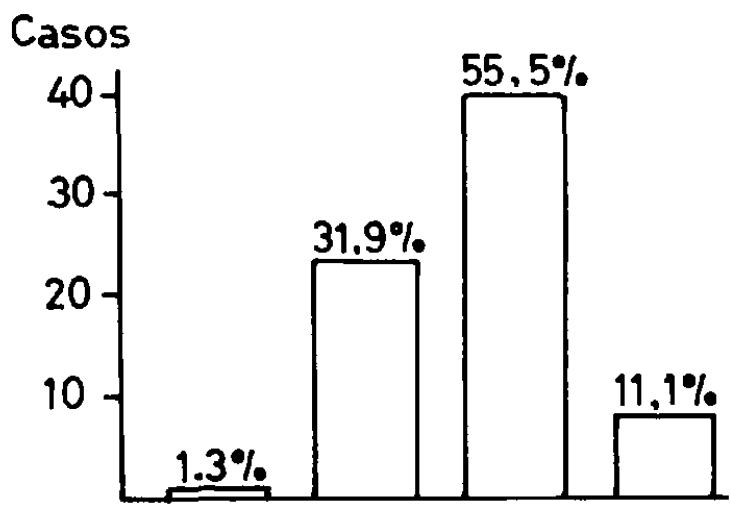

E.Inf. Obs.ER. E.R. Otros

Del análisis del cuadro clínico observamos Carditis en un $79.1 \%$, Poliartritis Migratoria en un $19.4 \%$ y Corea Reumática en un 5.5\%, Eritema Marginado en un caso con el 1.3\%. De los signos menores predominaron las Poliartralgias y la Fiebre. (Tabla 1.)
Tabla $N^{0} 1$

SIGNOS MAYORES Y MENORES

\begin{tabular}{|c|c|c|}
\hline SIGNOS MAYORES & $N^{\circ}$ & $\%$ \\
\hline $\begin{array}{l}\text { CARDITIS } \\
\text { POLIARTRITIS } \\
\text { COREA } \\
\text { ERITEMA MARGINADO } \\
\text { NODULOS S CUTANEOS }\end{array}$ & $\begin{array}{r}57 \\
14 \\
4 \\
1 \\
0\end{array}$ & $\begin{array}{rl}79 & 1 \\
19 & 4 \\
5 & 5 \\
1 & 3 \\
0 & 0\end{array}$ \\
\hline SIGNOS MENORES & No & $\%$ \\
\hline $\begin{array}{l}\text { POLIARTRALGIAS } \\
\text { FIEBRE }\end{array}$ & $\begin{array}{l}53 \\
42\end{array}$ & $\begin{array}{l}736 \\
583\end{array}$ \\
\hline
\end{tabular}

De los exámenes de laboratorio, la velocidad de sedimentación globular fue de $50 \mathrm{~mm}$. o más en la primera hora en 54 casos con un $74.9 \%$. (Gráfico 4.) En el hemograma se analizó la leucocitosis y la desviación a izquierda, encontrándose que el $49.2 \%$ de los pacientes tenían 10.000 leucocitos o más por $\mathrm{mm}^{3}$, y el $17.2 \%$ tenían más de 6 Baciliformes en la fórmula leucocitaria. (Gráfico 5.)

Gráfico $\mathrm{N}^{\circ} 4$

VALORES SEDIMENTACION GLOBULAR

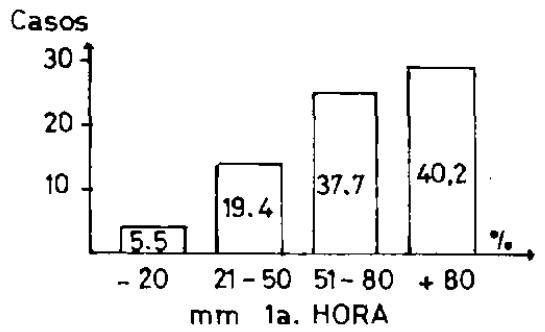

Grático $N^{\circ} 5$

LEUCOCITOSIS Y DESVIACION A IZDA. EN EL HEMOGRAMA

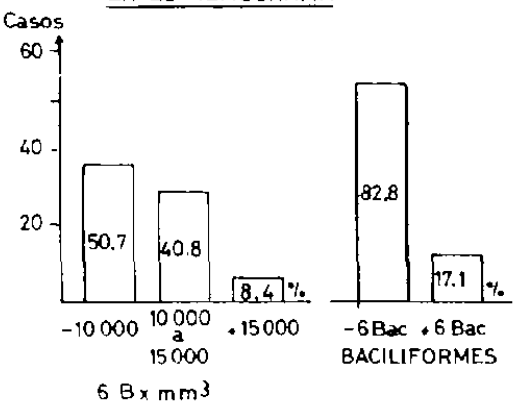


El título de A.S.O. se realizó en 28 casos y de éstos el $10.71 \%$ tuvo menos de $200 \mathrm{U}$. Todd, el $46.4 \%$ tuvo entre 200 y $500 \mathrm{U}$. Todd, el $28.5 \%$ entre 500 y $1.000 \mathrm{U}$. Todd, y el $14.2 \%$ curs 6 con más de 1.000 U. Todd. En total el $89.1 \%$ presentó más de 200 U. Todd. (Gráfico 6.)

\section{Grático № 6}

\section{IITULOS ASO INFORMADAS} 28 CASOS

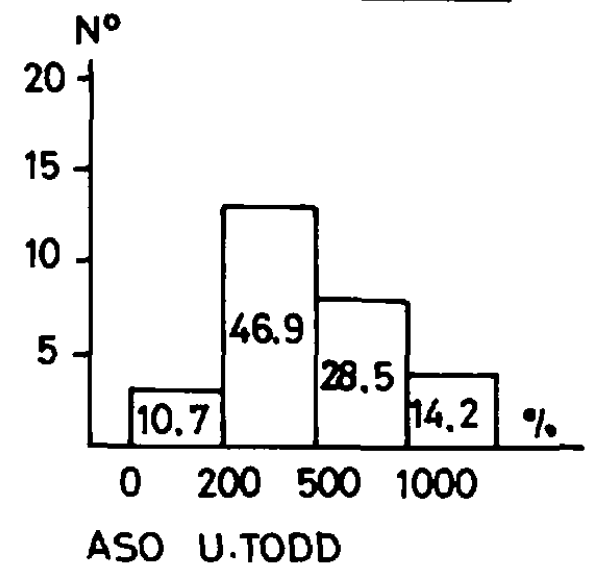

La radiografía de tórax se realizó en 41 casos, de éstos fue normal en 20 casos, observándose Cardiomegalia en 14 casos con un $34.1 \%$. (Gráfico 7.)

\section{Gráfico N.o 7}

\section{RX TORAX 41 CASOS}

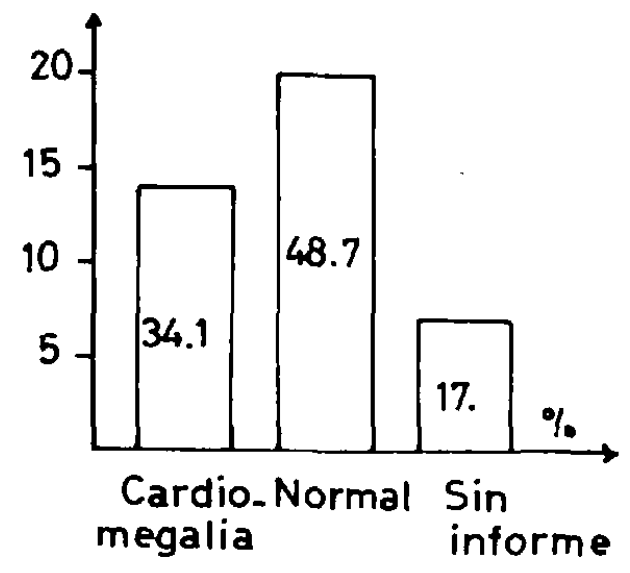

El Electrocardiograma se hizo en 54 casos y fue normal en 28 pacientes, el espacio P-R prolongado se observó en 5 casos con un $5 \%$. (Gráfico 8.)

Grático $N^{0} 8$

E.C.G. 54 CASOS NORMAL 28 CASOS $(51,85 \%)$

\section{Casos}

Anormales

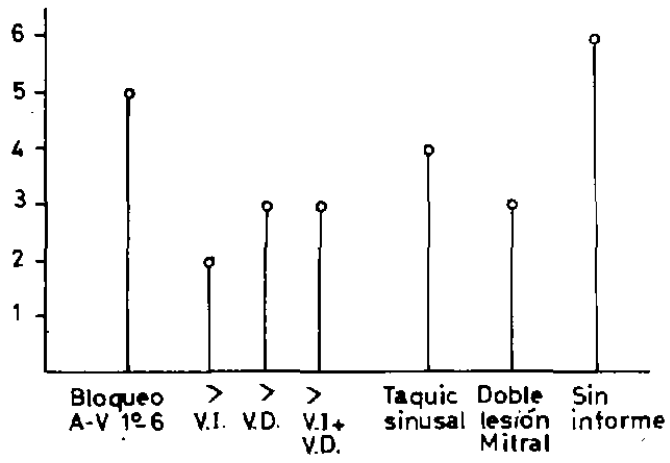

Del análisis del tratamiento efectuado y su relación con el número de recaídas y rebote, se observan 2 recaídas en casos con tratamiento esteroidal prolongado, tres recaídas y 1 rebote con tratamiento abreviado, 6 recaídas en aquellos casos tratados con Aspirina y 3 recaídas en casos con tratamiento no clasificable, con un total de 14 recaídas. (Tabla 2.)

Tabla No2

TRATAMIENTO EFECTUADO RECAIDAS Y REBOTE

\begin{tabular}{|l|c|c|c|c|c|c|}
\hline \multirow{2}{*}{ TRATAMIENTO } & \multicolumn{3}{|c|}{ FORMA DE E.R. } & Recald & Rebote \\
\cline { 2 - 7 } & Carditis & Artic.P. & Corea & Total & & \\
\hline CORTICOIDE PROLON & 12 & 1 & - & 13 & 2 & - \\
CORTICOIDE ABREV. & 22 & - & - & 22 & 3 & 1 \\
ASPIRINA & 17 & 10 & - & 27 & 6 & - \\
TRAT. NO CLASIFIC. & 5 & 1 & 4 & 10 & 3 & - \\
\hline TO T A L & 56 & 12 & 4 & 72 & 14 & 1 \\
\hline
\end{tabular}

De las secuelas valvulares observadas en 13 casos, 12 fueron de válvula Mitral con un $92.3 \%$ y un caso con lesión de válvula aórtica. (Gráfico 9.)

Gráfico No 9

SECUELAS VALVULARES

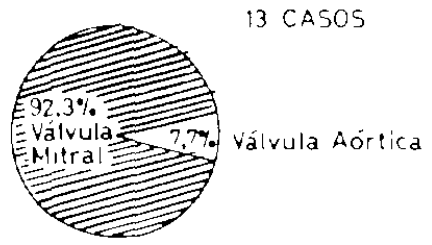


Se analizan las complicaciones y su relación con el tratamiento efectuado, encontrándose Insuficiencia Cardíaca en 6 casos, 3 casos sin lesión valvular en pacientes tratados con esquema prolongado, un caso de Insuficiencia Cardíaca más secuela valvular tratado con esquema prolongado y 2 casos de Insuficiencia Cardíaca más secuela valvular tratados con esquema abreviado. (Tabla 3.)

De las secuelas valvulares puras, 2 casos de 13 se trataron con esquema prolongado, 6 casos de 22 fueron tratados con esquema abreviado, un caso con Aspirina y un caso con tratamiento no clasificable.

El promedio de días de hospitalización con esquema abreviado fue de 27.42 días y con tratamiento prolongado de $\mathbf{4 2 . 2 3}$ días. (Gráfico 10.)

\section{Tabla $N^{\bullet} 3$}

COMPLICACIONES Y TRATAMIENTO EFECTUADO

\begin{tabular}{|l|c|c|c|}
\hline \multirow{2}{*}{ TRATAMIENTO } & \multicolumn{3}{|c|}{ COMPLICACIONES } \\
\cline { 2 - 4 } & \multicolumn{1}{|c|}{ I.C. } & LC. S.V. & S.V. \\
\hline CORTICOIDE PROLONG. & 3 & 1 & 2 \\
CORTICOIDE ABREVIAD. & - & 2 & 6 \\
ASPIRINA & - & - & 1 \\
TRAT. NO CLASIF. & - & - & 1 \\
\hline TOTAL & 3 & 3 & 10 \\
\hline
\end{tabular}

Gráłico $N^{\circ} 10$

RELACION DIAS HOSPITALIZACION Y TRATAMIENTO ESTEROIDAL

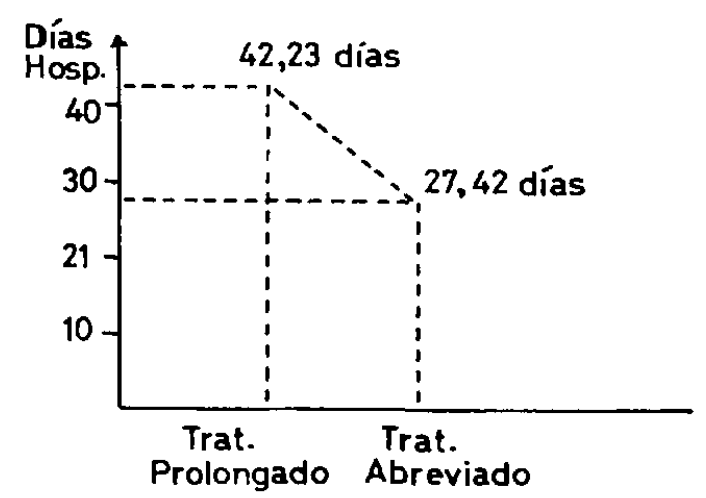

De las recaídas observadas, 12 casos $(85.7 \%)$ no tenían tratamiento profiláctico adecuado; y con profilaxis adecuada se observaron 2 casos $(14,3 \%)$, de los cuales un caso cumplía tratamien- to con Sulfadiazina por alergia a la Penicilina y el otro caso con Penicilina Benzatina cada 28 días. (Gráfico 11.)

El control posterior al alta se logró detectar sólo en 22 pacientes (33\%) y de éstos sólo 16 casos (22\%) tenían tratamiento profiláctico bien llevado. (Gráfico 11.)

Grático $N^{\circ} 11$

RELACION . CONTROL POSTERIOR, RECAIDAS Y TRATAMIENTO PROFILACTICO

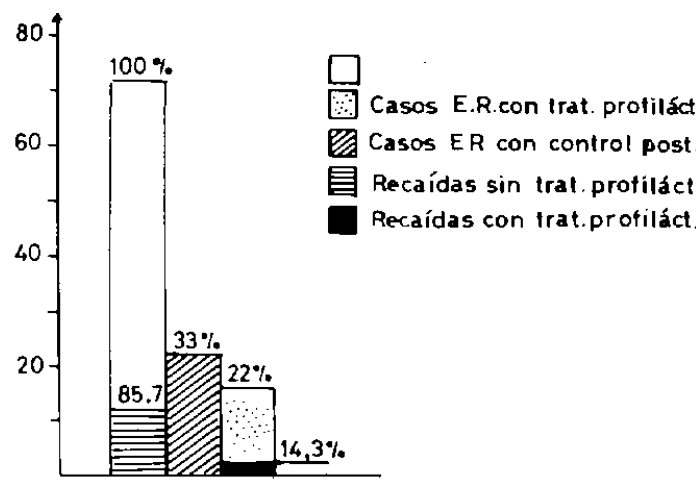

\section{COMENTARIO}

De las 150 fichas revisadas, sólo 72 correspondieron a Enfermedad Reumática, aplicando en forma estricta el criterio de Jones modificado.

La distribución por sexo, edad del primer brote, cuadro clínico y el compromiso valvular fueron similares a los observados por otros autores. $(3,4,5,6,7)$

El mayor porcentaje de recaídas se observó en niños tratados con salićlicos y en aquellos casos con tratamiento no clasificable, debiendo hacer notar que de nuestra casuística, 17 carditis fueron tratadas con Aspirina y hubo 5 casos con tratamiento no clasificable. No se observó diferencia significativa de recaídas en los casos tratados con esquema abreviado o prolongado. El rebote se presentó en un caso tratado con esquema abreviado, en el que fue necesario hacer una segunda cura esteroidal.

El promedio de días de hospitalización se reduce de $\mathbf{4 2 . 2 3}$ días a $\mathbf{2 7 . 4 2}$ días desde la introducción del esquema abreviado.

Sólo se pudo detectar el control posterior al alta en el $33 \%$ de los casos, lo que se puede atribuir a la no existencia de un programa preestablecido de control para el enfermo reumático en nuestra Región. Actualmente está en introduc- 
ción la tarjeta de profilaxis secundaria confeccionada en nuestro Servicio para el enfermo reumático del área de Talcahuano.

Sólo 2 niños con tratamiento profiláctico adecuado hicieron recaídas, uno de ellos en tratamiento con Sulfadiazina por alergia a la Penicilina y otro niño en tratamiento con Penicilina Benzatina cada 28 días.

C A R N E T

PRE VENCION Y CONTROL

\author{
E N F. R E U M T I C A
}

Nombre:

Nombre Madre:

No. Obs:

Domicilio:

Fecha Necimiento:

Edad:

Poli. Control:

Establ. Educacional:

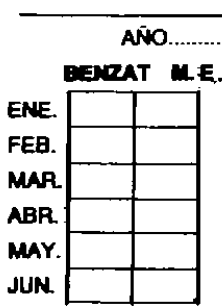

Fecha 1er. brote:

Menifeatación:

Clinicas:

Secueles:

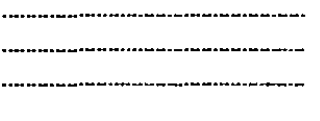

AÑO

\begin{tabular}{|c|c|}
\hline ENE & \\
\hline FEA. & \\
\hline MAR & \\
\hline ABA. & \\
\hline MAY & \\
\hline NW. & \\
\hline
\end{tabular}

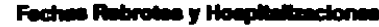
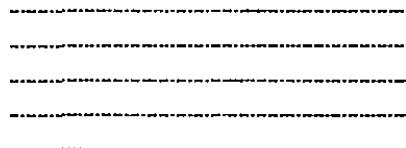

AÑo
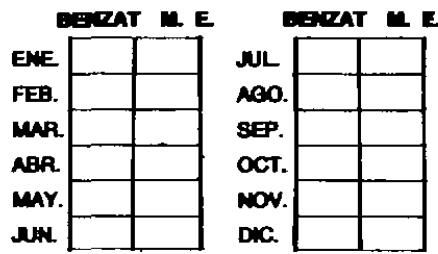
Se analizan los resultados obtenidos en la revisión de 72 fichas clínicas de Enfermedad Reumática en el Hospital Las Higueras de Talcahuano.

Se compara: complicaciones, secuelas valvulares, recaídas y promedio de días de hospitalización en relación con el tratamiento efectuado. Se observa que no hay diferencias significativas con el tratamiento abreviado o prolongado, salvo en la reducción de los días de hospitalización, la cual es muy notoria e iría en ayuda a la economía de nuestros Hospitales, sin que signifique algún riesgo para nuestros pacientes.

\section{SUMMARY}

After reviewing 72 clinical file records of Rheumatic Fever in Las Higueras Hospital.

The final results were analysed for comparison as follows: Complications, cardiac involvement, recurrences and average days-in-bed all in connection with therapeutic treatment.

No significative difference regarding a shortened or prolonged treatment is noticeable if we except an obvious decrease in the days-in-bed item, a decrease that means a relief to our Hospital budget but means no risk to the patient involved.
1 Sánchez, J., Philippi, O., Vicuña D.: Fiebre Reumática y Cardiopatía Reumática en Chile. R. Ch. Ped. 45, 4, 1974.

2 Medina, E. y Kaempfer, A.M.: Epidemiología de la Enfermedad Reumática en Chile. R. Med. Chile. 98: 646, 1970.

3 Vicuña, D., Saieh, M., Eimbcke, F. y Col. Evolución de la Carditis Reumatica tratada con un esquema abreviado de Prednisona. R. Ch. Ped. 45, 4, 1974.

4 Wilson, M.G. and Helper, H.N.: Effect of pituitary adrenocorticotropic hormone (ACTH) in Acute Rheumatic Carditis. J.A.M.A. 145: 135, 1951.

5 Wilson, M.G., and Lim, W. N.: Natural Course of active Rheumatic Carditis and evaluation of hormone therapy. J.A.M.A. 160: 1457, 1956.

6 Wilson, M.G. and Lim, W.N.: Short term Hormone Therapy. Its effects in Active Rheumatic Carditis of Variying Duration. New Engl. J. Med. 260: 802, 1959.

7 Wilson, M.G., Helper, H.N., Lubschez, R. Haim K., and Epstein, $N$.: Effects of short term administration of Corticotropin in Active Rheumatic Carditis. Ann. J. Dis. Child. 86: $131,1953$. 